\title{
Industry Clustering, Industry Specialization, and the Utilization Efficiency of Urban Land: Evidence from Prefectural-Level Cities in China
}

\author{
Zhangsheng Liu ${ }^{1,2, *}$, Deyong Song ${ }^{2}$, Yuanyuan Gong ${ }^{3}$ and Dongfang $\mathrm{Li}^{1}$ \\ 1 College of City Construction, Jiangxi Normal University, Nanchang 330022, China; \\ 2 School of Economics, Huazhong University of Science and Technology, Wuhan 430074, China; \\ dysong@hust.edu.cn (D. S.); d201577890@hust.edu.cn (D. L.) \\ 3 School of Economics, Henan University of Economics and Law, Zhengzhou 450046, China; \\ yygong@hust.edu.cn \\ * Correspondence: liuzs@hust.edu.cn; Tel.: +86-027-8754-3151
}

\begin{abstract}
In this paper, a land utilization efficiency evaluation model, which takes environmental loss into consideration, has been structured via taking advantage of the slack-based measure (SBM) model. Meanwhile, based on the panel data from 280 prefecture-level cities in China from 2003 to 2013, the paper thoroughly probed into, and discussed, the effect imposed by industry clustering and specialization on the utilization efficiency of urban land. Research results indicate several conclusions, as follows: (1) Taking environmental loss into account, the land utilization efficiency of prefecture-level cities in China is generally low. During the research period, the average value of the land utilization efficiency of prefecture-level cities in China is only 0.349, with, first, a declining trend, and then a rise. Geographically speaking, the land utilization efficiency presents a "depression in the center" phenomenon which means the land utilization efficiency of prefecture-level cities in the central China are relatively lower than in the east and west. Now, the difference among the urban land utilization efficiency in China significantly reflects the distinctions among Eastern, Western, and Central China. Moreover, the contribution degree of the difference of the land utilization efficiency among cities of central China to the aggregation difference shows an ascending momentum. Additionally, the relation between the population scale and land utilization efficiency in cities manifests as a U shape; (2) theoretically speaking, the relation between industry clustering and urban land utilization efficiency presents an inverted-U shape. However, this kind of relation is not significant in Western and Central China and medium-sized cities. Moreover, most of cities are still relatively far away from the inflection point or the critical value; and (3) the industry professional level has imposed a positive influence on urban land utilization efficiency. However, that influence is not significant in Eastern China and large cities. Consequently, strengthening the industry professional development of Western and Central China and small and medium-sized cities, facilitating diversified development of industries in Eastern China and large cities, and accelerating industrial clustering, all of these measures above will be conducive to improving urban land utilization efficiency in China.
\end{abstract}

Keywords: SBM model; industrial specialization; industrial clustering; urban land utilization efficiency

\section{Introduction}

The development path of new-style urbanization and industrialization is a crucial supporting point for continuous growth of China's economy. Urban land is the spatial carrier of the economy, society, and environment of cities [1]. However, blind and mindless urban expansion has also generated a series of issues for China in terms of land utilization, such as construction land increase and the threat of the warning limit of arable land, the tension of development land and regional 
land idleness, the dislocation of industrial layout, the waste of land resources, and so on. Therefore, seeking approaches of improving land utilization efficiency is significantly vital to new-style urbanization development in China.

Urban land is considered as the spatial carrier of industry clustering development. Moreover, industries are the source of power for urban development. Hence, facilitating the integrated development of urban cities and industries will help to effectively achieve sustainable development of the economy and society. Additionally, the integration of urban land and industries is also the process of industrial clustering, which presents two aspects, namely, the clustering of the total quantity of industries and the structure of industrial clustering (there are two forms of the structure of industrial clustering, namely, industrial specialization and diversification). In real life, it can be discovered that different cities with various industrial clustering, and their according clustering structures, will have significantly distinct land utilization efficiency. However, there are few theses discussing this phenomenon.

Concerned with the interactions and relations between industry clustering and urban economic growth and the environment, existing studies show that there are two different phases [2-3]. The first phase is where industry clustering is conducive to scaling the economy, closely linking related enterprises, which will contribute to making use of the learning effect, facilitate spillover of technology and knowledge, enhance economic increases, improve resource utilization efficiency, and reduce polluted emissions. Another phase will come when the degree of industry clustering exceeds the optimal urban economic scale. This situation will bring about a crowded effect and increase the consumption of raw materials which will hinder economic growth and exacerbate environmental pollution. Therefore, it is clear that there may exist similar development phases as above for the relation between industry clustering and urban land utilization efficiency.

Since the 1980s, with constant development of the new economic growth theory, urban economic scholars started to focus on the effect of industry clustering structure (namely, industry specialization and industry diversification) on the urban economy. Subsequently, two different points of view have been generated as follows [4-6]: On one hand, some scholars believe that local industry specialization will significantly facilitate knowledge spillover and enhance economic growth, and this point of view is considered as MAR spillover ${ }^{\circledR}$. On the other hand, others hold that industry diversification will be more conducive to knowledge spillover and economic growth, which is called Jacobs spillover. Therefore, it can be found that the industry clustering structure, via knowledge spillover, imposes influence on urban land utilization efficiency. However, there are few studies probing into which kind of knowledge spillover is more important, the one caused by industry specialization, or the one brought about by industry diversification.

In conclusion, there may be a non-linear relation between industry clustering and land utilization efficiency. Additionally, it has been discovered that the industry clustering structure, via knowledge spillover, imposes influence on urban land utilization efficiency. However, given the context of China's situation, the practical influence should be discussed further.

\section{Measurement of Land Utilization Efficiency}

\subsection{Measuring Methods of Land Utilization Efficiency}

In recent years, the Data Envelopment Analysis (DEA) method has been frequently adopted in

(1) MAR spillover comes from Marshall (1890), Arrow (1962) and Romer (1986, 1990)'s contribution and stresses the externalization of localization with the core idea of specialization spillover. 
plenty of research literature in order to discuss and research land utilization efficiency [7-10]. Through using DEA, the essential characteristics of land utilization have been fully taken into consideration, and land utilization has been considered as an input-output system. Meanwhile, there is no need to presume a certain production function for DEA, and there will be no influence from the dimensional unit; therefore, resulting errors caused by function setting and index weight setting can be avoided [11-13].

Currently, the studies employing DEA to evaluate the urban land utilization efficiency possess features as follows: At first, based on the perspective of input and output variables, land, capital, labor, etc., have been regarded as input elements. Meanwhile, GDP, industry production value, and so on have been considered as output variables. Secondly, focusing on research methods, it can be discovered that the BCC and CCR models of the DEA approach have been adopted in most studies [8-15], while the super-efficiency DEA [16] and bootstrap-DEA [17] approaches have been utilized in a few cases. However, during the process of industrialization and urbanization in China, due to the spread of extensive development patterns, tremendously fast economic growth has been achieved with the heavy costs of excessive resource consumption and environmental deterioration. Consequently, when land utilization efficiency is being evaluated, it is debatable, in terms of output, to only take GDP and industry production value into account, neglecting resources and environmental constraints. Thus, there is room for further improvement.

Scholars have made various attempts in order to make use of the DEA method to measure the economic efficiency considering undesirable output. In the existing literature, there are several methods which have been adopted in order to bring environmental factors into the economic efficiency model analysis; for instance, the method [18] which sets undesirable outputs as the input elements, the hyperbolic method [19], and the method [20] which takes undesirable outputs as output elements. Among those, the method which considers environmental emissions as undesirable outputs and then brings them into the production process has been widely applied [21]. For example, Chung et al. have incorporated polluted emissions into the production process and proposed the analysis model of environmental regulation behavior, which is based on a directional distance function, and this model preferably tackled the efficiency evaluation issue of undesirable output [22]. Nevertheless, this kind of directional distance function is a radial and oriented DEA model when it is applied to evaluate efficiency, which cannot completely take the slackness of the input and output into consideration. At the same time, it is necessary to make input-oriented or output-oriented choices; namely, a certain aspect, either output or input, may be ignored. Furthermore, there may be errors in the measured efficiency value [23]. In order to deal with the issue above, Tone has proposed a non-radial ad non-oriented slack-based measure (SBM) model. Therefore, in this paper, environmental loss has been included and considered in the evaluation system, and the SBM model has been adopted to measure the land utilization efficiency of each prefecture-level city. In this paper, each city has been regarded as a DMU (decision-making unit). It has been assumed that there are $m$ different kinds of input in every single DMU $x=\left(x_{1}, \cdots, x_{m}\right) \in R_{+}^{m}$, and it will generate $n$ different kinds of desirable output $y=\left(y_{1}, \cdots, y_{n}\right) \in R_{+}^{n}$ and $k$ different kinds of undesirable output $b=\left(b_{1}, \cdots, b_{k}\right) \in R_{+}^{k}$. Subsequently, the input and output value of province $j$ in period $t$ can be represented by $\left(x^{j, t}, y^{j, t}, b^{j, t}\right)$. Consequently, the production possibility set measuring the land utilization efficiency can be 
structured as follows:

$$
P^{t}\left(x^{t}\right)=\left\{\left(y^{t}, b^{t}\right) \mid \bar{x}_{j m}^{t} \geq \sum_{j=1}^{J} \lambda_{j}^{t} x_{j m}^{t}, \bar{y}_{j n}^{t} \leq \sum_{j=1}^{J} \lambda_{j}^{t} y_{j n}^{t}, \bar{b}_{j k}^{t} \geq \sum_{j=1}^{J} \lambda_{j}^{t} b_{j k}^{t}, \lambda_{j}^{t} \geq 0, \forall m, n, k\right\}
$$

Afterwards, based on the research from Tone, following SBM model can be structured:

$$
\rho^{*}=\min \frac{1-\frac{1}{m} \sum_{i=1}^{m} \frac{\bar{x}_{i}}{x_{i 0}}}{1+\frac{1}{n+k}\left(\sum_{r=1}^{n} \frac{\bar{y}_{r}}{y_{r 0}}+\sum_{l=1}^{k} \frac{\bar{b}_{l}}{b_{l 0}}\right)}
$$$$
\text { s.t. }\left\{\begin{array}{l}
x_{0}=\sum_{j=1, \neq 0}^{J} \lambda_{j} x+\bar{x}, \\
y_{0}=\sum_{j=1, \neq 0}^{J} \lambda_{j} y-\bar{y}, \\
b_{0}=\sum_{j=1, \neq 0}^{J} \lambda_{j} b+\bar{b}, \\
\bar{x} \geq 0, \bar{y} \geq 0, \bar{b} \geq 0, \lambda_{j} \geq 0
\end{array}\right.
$$

Especially, $\bar{x}, \bar{y}, \bar{b}$ are, respectively, the slacks quantity of input, desirable output, and undesirable output. $\lambda_{j}$ is the weight vector quantity; if the sum of $\lambda_{j}$ is equal to 1 , it signifies a variable return scale (VRS), otherwise it means a constant return scale (CRS). VRS has been selected in this paper given the law of scale return of land input and output. The larger the target function of $\rho^{*}$, the more efficient the evaluation unit is, and $0 \leq \rho^{*} \leq 1$. In addition, $\rho^{*}<1$ means that the evaluation unit is inefficient, and there is room for improvement of the output and input.

\subsection{Index selection and Data Processing}

Based on the statistical analysis principle of comparability and feasibility, thoroughly and comprehensively considering data availability, 280 prefecture-level cities from 2003 to 2013 have been selected for the research ${ }^{\circledR}$. The data mainly derives from the China City Statistical Yearbook and China Urban Construction Statistical Yearbook over the years, and it also comes from the statistical yearbook of every province, city, and municipality in the related years. Part of the missing data has been complemented by the interpolation method.

\footnotetext{
(2) This paper selects 280 cities except Chaohu, Hefei, Bijie, Tongren and Lhasa city as the research subjects in order to conduct feasible comparison. This is due to the reasons: (1) Chaohu city was adjusted as county-level city and was administrated by Hefei city, where may have the problem of overlapping data. Therefore, data of Chaohu and Hefei city are excluded; (2) statistical caliber are not consist in Bijie city and Tongren city as they were set up in 2011; and (3) Lhasa is excluded as a lack of data.
} 


\subsubsection{Input Factors}

The classical production function manifests that the basic input of production includes capital and the labor force. At the same time, the land utilization is the spatial carrier of the urban economy. Therefore, in this paper, input elements contain the labor force, land, and capital stock. Especially, the labor force is represented as the number of unit employees in cities at the end of the year, and land is represented as the size of urban built-up area. Moreover, capital stock is calculated referring to the method of Zhang [24]. Commodity prices can be replaced by the price index of the province, city, and municipality where the sample city is located: (Investment's deflating price index $=0.45 \times$ FAIPI (Fixed Assets Investment Price Index) $+0.55 \times$ CPI (Consumer Price Index)). In addition, it has been assumed that the growth rate of capital stock and the increasing rate of actual investment is the same in order to calculate the capital stock of the base year (2003 is the base period).

\subsubsection{Output Factors}

Desirable output: GDP has been selected as the indicator measuring economic output. Meanwhile, the CPI of the province, city, and municipality where the sample city is located has been utilized to be deflated (adopting the year of 2000 as the base period).

Undesirable output: Frequently-mentioned negative or bad output contains the emission amount of three industrial wastes [25]: sulfur dioxide [26], chemical oxygen demand (COD) [27], and carbon dioxide [28]. Moreover, in order to avoid the negative output elements being excessively singular, in this paper, the emission sum of urban industrial waste water and industrial sulfur dioxide have been taken as the undesirable outputs.

\section{The Characteristics of Chinese Urban Land Utilization Efficiency}

\subsection{General Characteristics}

According to the discussions above, the general situation of the land utilization efficiency of Chinese prefecture-level cities (2004-2013) has been presented in Figure 1. To be detailed, from 2003 to 2013, the Chinese urban land utilization efficiency is generally low. Moreover, the average efficiency value of 280 prefecture-level cities is 0.349 . That value is below the result of Wu et al., which is 0.524 , and also slightly lower than the result of Zhang, which is 0.371 , and the result of Liang et al., which is 0.414 . Primary reason for the difference may be that the research periods of the literature above are different and the environmental restrictions have not been taken into consideration. From the perspective of the distribution intervals of the land utilization efficiency, the efficiency values of most cities are between 0.25 0.5 (there are, in total, 188 cities, contributing $67.1 \%$ of the aggregate). Additionally, there are 48 cities whose land utilization efficiency values are lower than 0.25 , accounting for $17.1 \%$, and $9.3 \%$ cities range from 0.5 to 0.75 . Moreover, there are only 18 cities whose values are larger than 0.75 , contributing $6.4 \%$ of the aggregate. The results above signify that the urban land utilization efficiency of most cities in China is a medium-low level. Theoretically speaking, under the given condition of no limit on the output, there is still land saving potential of $65.1 \%$ for prefecture-level cities in China. Consequently, abandoning blind and aimless expansion and enhancing population agglomeration as well as industry specialization will be the important working direction of land utilization efficiency improvement in China. From the perspective of time-order characteristics, from 2003 to 2013, the land utilization efficiency of Chinese prefecture-level cities generally showed, first, decreasing momentum, and then increasing. All of the minimum values are in 2005. This is primarily due to the fact that, since 2006, China has 
strengthened environmental governance and gradually transformed its developmental ideas, turning from an extensive economic developmental model into the development of connotation, further enhancing the improvement of the land utilization efficiency under environmental restrictions.

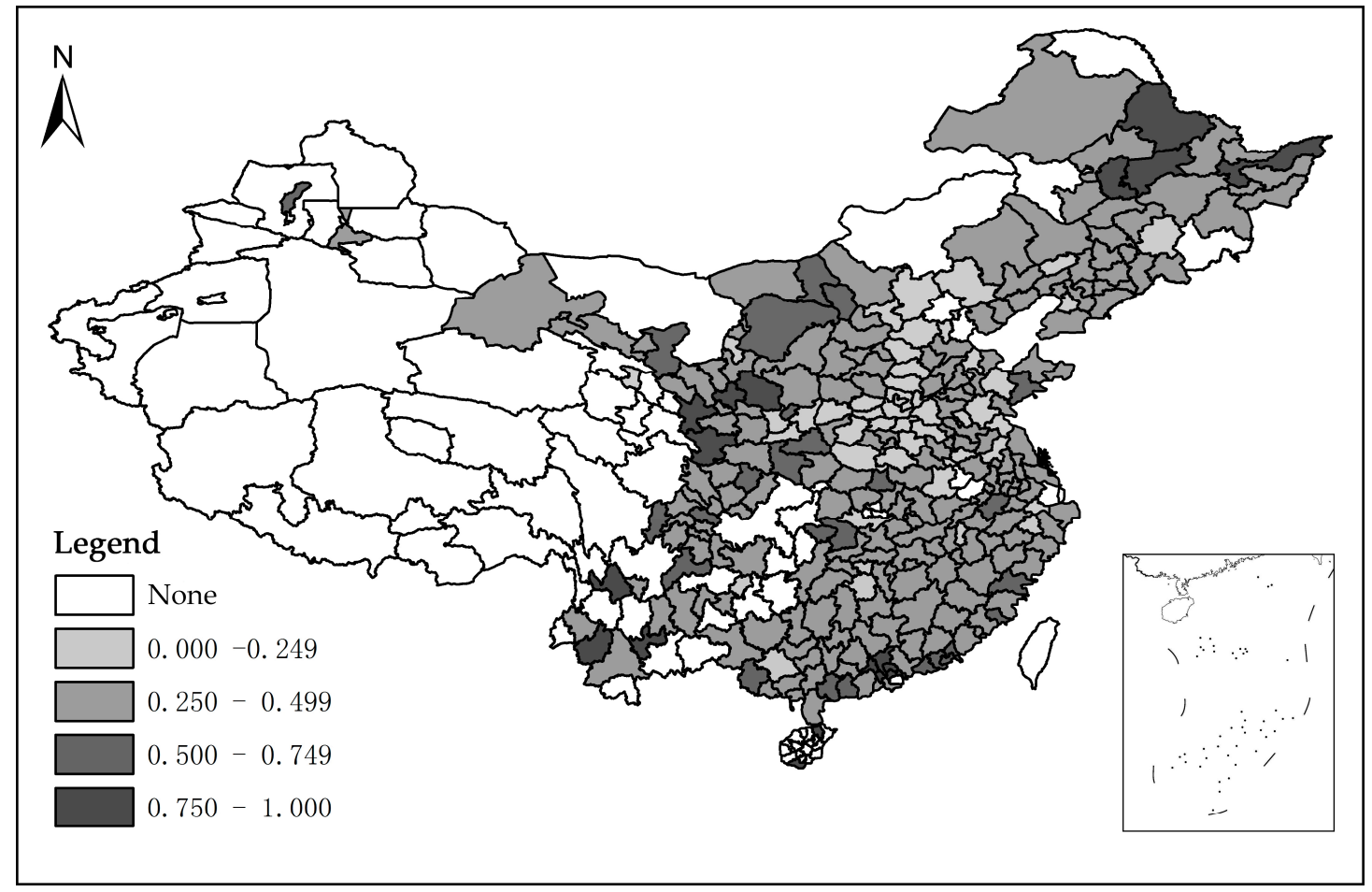

Figure 1. Spatial distribution of the land utilization efficiency of 280 prefecture-level cities in China (2004-2013).

\subsection{Spatial Characteristics}

From the perspective of the individual differences of cities, the lowest average value of efficiency is only 0.162 , which is less than $1 / 6$ of the frontier efficiency value. Figure 1 shows the spatial distribution of the land utilization efficiency of 280 prefecture-level cities in China. Through Figure 1, it can be determined that there are relatively large differences in the spatial distribution of the land utilization efficiency of prefecture-level cities of China. Moreover, to begin with, the land utilization efficiencies of cities in Eastern China are higher than the ones in Central and Western China. Meanwhile, the land utilization efficiencies of cities in Western China are higher than the ones in Central China. This phenomenon also indicates that, geographically speaking, the land utilization efficiency of cities in China presents a "depression in the center" phenomenon, which means the land utilization efficiency of prefecture-level cities in Central China are relatively lower than in the east and west. The main reason leading to this phenomenon is as follows: along with the economic developmental model transformation of Eastern China, the according enhancement of environmental awareness and the strengthening of environmental restrictions and regulations, subsequently, some industries of high energy consumption, high polluted emissions, and low efficiency are gradually transferring from Eastern China to Central and Western China. Particularly, Central China is the preferred area for industries to transfer to. In addition, during the past period, the amount of industries transferring to central China from the eastern part is larger than from western China. Therefore, the factors above brought about the "depression in the center" phenomenon, given the condition of undesirable output. This also further suggests that there is a 
more prominent contradiction between economic development and sustainable development of resources and environment in Central China.

\subsection{Regional Characteristics}

In order to research and observe the characteristics of regional difference of Chinese urban land utilization efficiency under the environmental restrictions, the Theil coefficients of the land utilization efficiency has been calculated and, furthermore, the difference among eastern, central, and western areas of China, as well as the difference inside cities of different areas, and their according contribution to the total difference, has been calculated. The results are shown in Table 1.

Table 1. Theil coefficient and its contribution rate.

\begin{tabular}{|c|c|c|c|c|c|c|c|c|c|c|c|}
\hline \multirow{2}{*}{ Year } & \multicolumn{9}{|c|}{ Theil Coefficient } & \multicolumn{6}{c|}{ Contribution Rate } \\
\cline { 2 - 12 } & Nation & East & Center & West & $\begin{array}{c}\text { Inside } \\
\text { Areas }\end{array}$ & $\begin{array}{c}\text { Among } \\
\text { Areas }\end{array}$ & East & Center & West & $\begin{array}{c}\text { Inside } \\
\text { Areas }\end{array}$ & $\begin{array}{c}\text { Among } \\
\text { Areas }\end{array}$ \\
\hline 2004 & 0.1399 & 0.1394 & 0.1275 & 0.1351 & 0.1339 & 0.0059 & 0.3489 & 0.3224 & 0.2863 & 0.9576 & 0.0424 \\
\hline 2005 & 0.1536 & 0.1839 & 0.1138 & 0.1474 & 0.1483 & 0.0054 & 0.4189 & 0.2618 & 0.2844 & 0.9651 & 0.0349 \\
\hline 2006 & 0.1423 & 0.1308 & 0.1191 & 0.1540 & 0.1335 & 0.0088 & 0.3217 & 0.2958 & 0.3207 & 0.9383 & 0.0617 \\
\hline 2007 & 0.1163 & 0.1008 & 0.1119 & 0.1219 & 0.1110 & 0.0053 & 0.3033 & 0.3400 & 0.3107 & 0.9541 & 0.0459 \\
\hline 2008 & 0.1209 & 0.1290 & 0.0912 & 0.1205 & 0.1131 & 0.0078 & 0.3736 & 0.2667 & 0.2955 & 0.9359 & 0.0641 \\
\hline 2009 & 0.1186 & 0.1286 & 0.0893 & 0.1147 & 0.1106 & 0.0080 & 0.3797 & 0.2663 & 0.2868 & 0.9327 & 0.0673 \\
\hline 2010 & 0.1322 & 0.1422 & 0.1183 & 0.1179 & 0.1265 & 0.0056 & 0.3765 & 0.3163 & 0.2645 & 0.9573 & 0.0427 \\
\hline 2011 & 0.1363 & 0.1367 & 0.1134 & 0.1256 & 0.1251 & 0.0112 & 0.3508 & 0.2941 & 0.2730 & 0.9178 & 0.0822 \\
\hline 2012 & 0.1247 & 0.1180 & 0.1219 & 0.1105 & 0.1171 & 0.0075 & 0.3313 & 0.3456 & 0.2628 & 0.9396 & 0.0604 \\
\hline 2013 & 0.0887 & 0.0811 & 0.0963 & 0.0848 & 0.0876 & 0.0012 & 0.3199 & 0.3837 & 0.2834 & 0.9870 & 0.0130 \\
\hline
\end{tabular}

The Theil coefficients across the nation in Table 1 indicate that there are relatively significant characteristics of differences among Chinese urban cities in terms of land utilization efficiency under the environmental restrictions. The difference of the land utilization efficiency is the largest in 2005 and is the smallest in 2013. From the perspective of the variations of the Theil coefficients, from 2004 to 2013, the differences of Chinese urban land utilization efficiency generally present a fluctuating, falling trend. Two troughs appear in 2009 and 2013. This phenomenon indicates that the gap of Chinese urban land utilization efficiency under the environmental restrictions is narrowing.

From the perspective of the structural decomposition of the Theil coefficients, the Theil coefficients among different areas are generally stable. However, variations of the Theil coefficients inside certain areas present a fluctuating, decreasing trend. Furthermore, its according troughs appeared in 2009 and 2013. The minimum value of the Theil coefficient inside certain areas appeared in 2013 and the maximum value appeared in 2005 which is basically in line with the law of the national Theil coefficients. From 2004 to 2013, the Theil coefficients inside certain areas (with an average value of 0.1207 ) were larger than those among different areas (with an average value of 0.0067). All of the results above show that the main difference of Chinese urban land utilization efficiency is the difference inside certain areas. When the areas have been split into three parts, in terms of the area differences, it can be determined that the largest is the eastern area, followed by the western area and, finally, is the central area.

From the perspective of the contribution rate of Theil coefficients of the three major areas of China, over the years the contribution rates of Theil coefficients inside certain areas are all over 90\%, which indicates that the difference of land utilization efficiency inside certain areas is the primary 
aspect of the difference of Chinese urban land utilization efficiency. The structural decomposition of the difference inside certain areas shows that the contribution rate of eastern areas to the total difference is around $35 \%$, for the central areas it is approaching $31 \%$, and for the western areas it is about $29 \%$. However, generally speaking, the contribution rate of eastern areas presents a fluctuating decreasing trend, and central areas act relatively stably, while western areas show an increasing trend.

\subsection{Analysis of Scaling Difference}

According to The State Council's Notice on City Scale Classification Standard Adjustment, which has been issued since 2014, the prefecture-level cities in China have been classified into three categories (based on the total population number of each city at the end of 2013), namely, small-sized cities (with an urban permanent population under 500,000), medium-sized cities (with an urban permanent population between 500,000 and 1,000,000), and large-sized cities (with an urban permanent population over 1,000,000). The distribution of the average land utilization efficiency of cities at different scales from 2004 to 2013 can be seen in Table 2.

Table 2. The differences of the average value of land utilization efficiency of different scale cities from 280 prefecture-cities from 2004 to 2013.

\begin{tabular}{|c|c|c|c|c|c|c|c|c|}
\hline Scale of cities & $\begin{array}{c}\text { Number } \\
\text { of cities }\end{array}$ & $\begin{array}{c}\text { Average } \\
\text { Value }\end{array}$ & $\begin{array}{c}\text { Standard } \\
\text { Deviation }\end{array}$ & Median & $\begin{array}{c}\text { Minimum } \\
\text { Value }\end{array}$ & $\begin{array}{c}\text { Quartile } \\
(\mathrm{Q} 1)\end{array}$ & $\begin{array}{c}\text { Quartile } \\
(\mathrm{Q} 3)\end{array}$ & $\begin{array}{c}\text { Maximum } \\
\text { Value }\end{array}$ \\
\hline $\begin{array}{c}\text { Large-sized } \\
\text { cities }\end{array}$ & 127 & 0.339 & 0.178 & 0.321 & 0.172 & 0.257 & 0.418 & 1.000 \\
\hline $\begin{array}{c}\text { Medium-size } \\
\text { d cities }\end{array}$ & 104 & 0.331 & 0.160 & 0.316 & 0.162 & 0.263 & 0.368 & 1.000 \\
\hline $\begin{array}{c}\text { Small-sized } \\
\text { cities }\end{array}$ & 49 & 0.424 & 0.220 & 0.381 & 0.210 & 0.305 & 0.492 & 1.000 \\
\hline
\end{tabular}

Based on the data in Table 2, it can be determined that, for the prefecture-level cities in China, from 2004 to 2013, the average values of the land utilization efficiency of large-sized cities, medium-sized cities, and small-sized cities are, respectively, 0.370, 0.355, and 0.465, which indicates that the relation between the scale of cities and their land utilization efficiency is U-shaped. At the same time, this law is similar with the research conclusions of $\mathrm{Wu}$ [9]. From the perspective of land utilization efficiency distribution, although there are few large- and medium-sized cities with relatively high land utilization efficiency, the land utilization efficiency of most cities are extremely low, which reduces the general level of land utilization efficiency. Moreover, small-sized cities possess significant advantages in terms of land utilization efficiency compared with other cities. Consequently, promoting the development of small-sized cities will be conducive to improving Chinese urban land utilization efficiency.

\section{Empirical Analysis}

\subsection{Empirical Model and Variables}

\section{(1) Empirical Model}

In order to inspect and research the concrete influence of industry clustering and its according structures on land utilization efficiency, based on the existing research literature and given that there may be a non-linear relation between industry clustering, urban land utilization efficiency, 
and the interactive relation between industry specialization and industry diversification ${ }^{\circledR}$, the quadratic term of the industry clustering level has been introduced in this paper. Moreover, an empirical model, as follows, has been formulated, taking the industry specialization as the proxy variable of the industry clustering structure.

$$
T E X_{i t}=\alpha_{0}+\alpha_{1} J J_{i t}+\alpha_{2} J J_{i t}^{2}+\alpha_{3} S S_{i t}+\alpha_{4} X_{i t}+u_{i t}
$$

In the equation above, the explained variable $T E X$ is the land utilization efficiency. Since the efficiency values which have been measured are between 0 and 1 , and for the convenience of model evaluation, the value of the measured efficiency has been extended 10 times during the empirical process. Meanwhile, the explaining variable $J J$ is the industry clustering level and $J J^{2}$ is the quadratic term of the industry clustering level. $S S$ is the index of industry specialization and $X$ is the control variable including the urban infrastructure situation, urbanization level, and environmental regulation, etc. $u_{i t}$ is the random disturbance term.

\section{(2) Variables Selection}

$J J$ is the industry clustering level. The industry clustering refers to the centralizing of industries in certain spatial areas. Ciccone and Hall believe that the degree of industry clustering can be comprehensively reflected by the internal economic density of cities. Based on that opinion, in this paper, the calculation results of the unchanged price output of each prefecture-level city of the year (GDP), being divided by the built-up area size, have been used to represent the industry clustering level $\left(10,000\right.$ yuan/ $\left.\mathrm{km}^{2}\right)$.

$S S$ is the industry specialization level. In the existing research literature, concerning the measurement of the urban industry specialization, the approaches of Duranton and Puga [29] have frequently been referred to, namely, the professional Gini coefficient. Therefore, in this paper, the index of urban industry specialization has been defined as follows:

$$
S S_{i}=\frac{1}{2} \sum_{j}\left|p_{i j}-p_{j}\right|
$$

In particular, $P_{i j}=L_{i j} / L_{i}$, where $L_{i j}$ represents employment figure of the $j$ industry of $i$ city, and $L_{i}$ represents the non-agricultural employment figure of city $i . P_{i j}$ refers to the employment proportion of $j$ industry contributing in $i$ city. $P_{j}$ refers to the employment proportion of $j$ industry in all prefecture-level cities. The larger $S S_{i}$ is, the higher the city's industry specialization level, while the smaller $S S_{i}$ is, the higher the city's industry diversification level.

\footnotetext{
(3) As two aspects of one issue, in some degree, industry specialization and diversification share the same essence. The single main difference lies on the index setting. Industry diversification will be accordingly examined after the analysis of industry specialization being exercised. Hence, analysis related with industry diversification has been omitted in this paper.
} 
The urban infrastructure situation is represented by road area per capita $\left(\mathrm{m}^{2}\right)$, and the urbanization level is referred to as the urban population density $\left(10,000\right.$ people $\left./ \mathrm{km}^{2}\right)$. In this paper, the industrial waste water amount per GDP and the varying rate of sulfur dioxide emission have been used to measure the environmental regulation. In addition, the approaches from Sun and Wang's research [30] have been adopted to measure the comprehensive indices. Moreover, according to the research of Wang and Liu [31], the linear term, quadratic term and cubic term of environmental regulation have been included in the model.

In order to match with the urban land utilization efficiency, all of the variables above have been selected from the statistical data of 280 prefecture-level cities from 2003 to 2013. The statistical data derives from China City Statistical Yearbook and China Urban Construction Statistical Yearbook over the years, and it also comes from the statistical yearbook of each province, city, and autonomous region in related years.

\subsection{Empirical Results Analysis of Total Samples}

The evaluation approaches of the panel data generally contain fixed effect and random effect regression. Since, within a relatively short period, the cross-section sample of the data selected in this thesis is relatively large, the fixed effect model will be more suitable. Meanwhile, a Hausman test also manifests that the original assumption of random effect has been rejected with a significance level of $1 \%$. In the total samples, via the stepwise regression treatment to Equation (3), and the variables mentioned above having been respectively evaluated, the author expects to probe the relation between industry clustering, industry specialization, and land utilization efficiency. The evaluation results can be found in table 3 .

Table 3. The evaluation results of total samples.

\begin{tabular}{|c|c|c|c|c|}
\hline Explaining Variables & $(1)$ & $(2)$ & $(3)$ & $(4)$ \\
\hline Industry Clustering & $\begin{array}{c}0.371^{* * *} \\
(14.58)\end{array}$ & & $\begin{array}{c}0.359^{* * *} \\
(14.09)\end{array}$ & $\begin{array}{c}0.318^{* * *} \\
(11.97)\end{array}$ \\
\hline The Quadratic Term of Industry Clustering & $\begin{array}{c}-0.00649^{* * *} \\
(-9.74)\end{array}$ & & $\begin{array}{c}-0.00630^{* * *} \\
(-9.47)\end{array}$ & $\begin{array}{c}-0.00571^{* * * *} \\
(-8.49)\end{array}$ \\
\hline Industry Specialization & & $\begin{array}{c}3.412^{* * *} \\
(5.90)\end{array}$ & $\begin{array}{c}2.525^{* * *} \\
(4.52)\end{array}$ & $\begin{array}{c}2.556^{* * *} \\
(4.60)\end{array}$ \\
\hline Infrastructure Situation & & & & $\begin{array}{c}0.0100^{*} \\
(1.69)\end{array}$ \\
\hline Urbanization Level & & & & $\begin{array}{c}0.0824^{* *} \\
(2.21)\end{array}$ \\
\hline Environmental regulation & & & & $\begin{array}{c}-2.327^{* * *} \\
(-3.95)\end{array}$ \\
\hline The Quadratic Term of Environmental regulation & & & & $\begin{array}{c}2.749^{* * *} \\
(2.66)\end{array}$ \\
\hline The Cubic Term of Environmental regulation & & & & $\begin{array}{c}-1.217^{* *} \\
(-2.54)\end{array}$ \\
\hline Constant Term & $\begin{array}{c}2.546^{* * *} \\
(26.63)\end{array}$ & $\begin{array}{c}3.059^{* * *} \\
(20.35)\end{array}$ & $\begin{array}{c}1.942^{* * *} \\
(11.83)\end{array}$ & $\begin{array}{c}2.300^{* * *} \\
(11.31)\end{array}$ \\
\hline Number of Sections & 280 & 280 & 280 & 280 \\
\hline Observed Value & 2800 & 2800 & 2800 & 2800 \\
\hline Hausman Test & $\begin{array}{c}15.96 \\
{[0.0012]}\end{array}$ & $\begin{array}{c}10.38 \\
{[0.0056]}\end{array}$ & $\begin{array}{c}37.06 \\
{[0.0000]}\end{array}$ & $\begin{array}{c}67.74 \\
{[0.0000]}\end{array}$ \\
\hline
\end{tabular}

Notes: ${ }^{* * *},{ }^{* *}$, and * respectively represent the evaluation values being significant at the level of $1 \%, 5 \%$, and $10 \%$. The values in the round brackets are $t$ statistical amounts under the robust standard errors, and the values in the square brackets are $p$-values.

Table 3 reports the evaluation results of the fixed effect model under different kinds of variable combinations. It has been discovered that the significance and influencing direction of industry clustering and industry specialization's effects on urban land utilization efficiency are almost the 
same. At the same time, there are several points concluded from Table 3.

Firstly, the coefficient of the linear term of industry clustering is positive, while the coefficient of the quadratic term is negative. Meanwhile, they are all significant at the $1 \%$ level in different models, which indicates that, theoretically speaking, there is a non-linear relation between industry clustering and the urban land utilization efficiency. The fact that the quadratic term is significantly negative indicates that the increase of the industry clustering level is conducive to knowledge spillover and, further, to increasing the land utilization efficiency when it has not reached the critical value. However, when the industry clustering level reaches and surpasses the critical value, the knowledge spillover effect will be weakened and, further, it will lead to the decrease of the land utilization efficiency. However, the coefficient of the quadratic term is relatively small, at 0.00571 , and the total critical value is $278,740 \mathrm{RMB} / \mathrm{km}^{2}$. In 2013, among the 280 prefecture-level cities in China, the average value of industry clustering was 5.382, its median was 4.772 , the minimum value was 0.790 , the quartile (Q1) was 3.354, and the quartile (Q3) was 6.324. In all of the cities, only Dongguan's and Foshan's industry clustering levels surpassed the critical value, which indicates that, for the majority of cites, industry clustering can significantly increase the land utilization efficiency.

Secondly, the regression coefficient of industry specialization is positive with a significance level of $1 \%$. This manifests that, from a general perspective, the increase of industry specialization level is conducive to improving the urban land utilization efficiency. In other words, the industry clustering structure in China currently presents as MAR spillover, namely, the knowledge spillover brought about by the industry specialization level improvement.

Thirdly, the regression coefficient of the infrastructure situation is 0.01 , which is significant at the level of $10 \%$. Therefore, generally speaking, the completion and improvement of urban infrastructures is beneficial to increasing the urban land utilization efficiency. In addition, the regression coefficient of the urbanization level is 0.0824 , which is significant at the level of $5 \%$, and this shows that active enhancement of new-style and human-centered urbanization will be conducive to improving the urban land utilization efficiency.

Fourthly, the first term coefficient of environmental regulation is -2.327 , the according quadratic term coefficient is 2.749 , and the cubic term coefficient is -1.217 , which are, respectively, significant at the levels of $1 \%, 1 \%$, and $5 \%$. These results show that the relation between environmental regulation and the land utilization efficiency is in line with an inverted N-shaped relation, and this conclusion is similar with the research results of Wang and Liu [31]. Namely, when the environmental regulation is relatively weak, social and environmental costs will be reduced and, accordingly, innovative motivation will be weakened, which will further decrease the urban land utilization efficiency. However, when the environmental regulation or restriction degree sits in a reasonable range, the social innovation will be promoted and this will further increase the urban land utilization efficiency. However, when the environmental regulation degree exceeds the affordable limit of society, the land utilization efficiency will also be reduced. Consequently, reasonable and suitable degrees of environmental regulation will be conducive to improving the urban land utilization efficiency.

\subsection{Empirical Results Analysis for Cities from Various Areas at Different Scales}

In China, there exist relatively large gaps in terms of the development situation among cities from different areas and at different scales. In order to observe and inspect these two types of differences, the author further exercises evaluations, respectively. The results are shown in Table 4. 
Table 4. Evaluation results of cities from different areas and at different scales.

\begin{tabular}{|c|c|c|c|c|c|c|}
\hline \multirow{2}{*}{$\begin{array}{l}\text { Explaining } \\
\text { Variables }\end{array}$} & \multicolumn{3}{|c|}{ Areas Types } & \multicolumn{3}{|c|}{ Scales Types } \\
\hline & East & Center & West & Small & Medium & Big \\
\hline $\begin{array}{l}\text { Industry } \\
\text { Clustering }\end{array}$ & $\begin{array}{c}0.181^{* * *} \\
(4.63)\end{array}$ & $\begin{array}{c}0.415^{* * *} \\
(3.72)\end{array}$ & $\begin{array}{c}0.446^{* * *} \\
(4.91)\end{array}$ & $\begin{array}{c}0.777^{* * *} \\
(6.09)\end{array}$ & $\begin{array}{c}0.373^{* * *} \\
(4.28)\end{array}$ & $\begin{array}{c}0.250^{* * *} \\
(6.52)\end{array}$ \\
\hline $\begin{array}{c}\text { The Quadratic } \\
\text { Term of } \\
\text { Industry } \\
\text { Clustering }\end{array}$ & $\begin{array}{c}-0.00337^{* * *} \\
(-3.98)\end{array}$ & $\begin{array}{c}-0.00363 \\
(-0.33)\end{array}$ & $\begin{array}{l}-0.0105 \\
(-1.18)\end{array}$ & $\begin{array}{c}-0.0321^{* * *} \\
(-2.79)\end{array}$ & $\begin{array}{c}-0.00399 \\
(-0.45)\end{array}$ & $\begin{array}{c}-0.00422^{* * *} \\
(-4.96)\end{array}$ \\
\hline $\begin{array}{c}\text { Industry } \\
\text { specialization }\end{array}$ & $\begin{array}{l}0.971 \\
(1.07) \\
\end{array}$ & $\begin{array}{c}2.690^{* * *} \\
(3.05)\end{array}$ & $\begin{array}{c}4.964^{* * *} \\
(4.44)\end{array}$ & $\begin{array}{c}5.628^{* * *} \\
(4.07)\end{array}$ & $\begin{array}{c}1.882^{* * *} \\
(2.67)\end{array}$ & $\begin{array}{l}1.020 \\
(1.04) \\
\end{array}$ \\
\hline $\begin{array}{c}\text { Infrastructure } \\
\text { Situation } \\
\end{array}$ & $\begin{array}{l}-0.0127 \\
(-1.37) \\
\end{array}$ & $\begin{array}{c}0.00656 \\
(0.53) \\
\end{array}$ & $\begin{array}{c}0.0419^{* * *} \\
(4.13)\end{array}$ & $\begin{array}{c}0.0425^{* * *} \\
(3.94)\end{array}$ & $\begin{array}{c}-0.00275 \\
(-0.26) \\
\end{array}$ & $\begin{array}{c}-0.0386^{* * *} \\
(-3.74) \\
\end{array}$ \\
\hline $\begin{array}{c}\text { Urbanization } \\
\text { Level }\end{array}$ & $\begin{array}{c}0.518^{* * *} \\
(4.61)\end{array}$ & $\begin{array}{l}0.0289 \\
(0.24) \\
\end{array}$ & $\begin{array}{c}0.0356 \\
(0.85) \\
\end{array}$ & $\begin{array}{c}-0.0356 \\
(-0.25)\end{array}$ & $\begin{array}{l}0.0927 \\
(1.10) \\
\end{array}$ & $\begin{array}{c}0.0734^{*} \\
(1.69)\end{array}$ \\
\hline $\begin{array}{c}\text { Environmenta } \\
1 \text { Regulation }\end{array}$ & $\begin{array}{c}-3.042^{* * *} \\
(-3.11)\end{array}$ & $\begin{array}{l}-1.631 \\
(-1.57)\end{array}$ & $\begin{array}{c}-3.472^{* * *} \\
(-2.86)\end{array}$ & $\begin{array}{l}-1.056 \\
(-0.72)\end{array}$ & $\begin{array}{c}-3.854^{* * *} \\
(-3.84)\end{array}$ & $\begin{array}{c}-2.494^{* * *} \\
(-2.64)\end{array}$ \\
\hline $\begin{array}{l}\text { The Quadratic } \\
\text { Term of } \\
\text { Environmenta } \\
\text { 1 Regulation }\end{array}$ & $3.817^{* *}$ & $\begin{array}{l}1.361 \\
(0.73)\end{array}$ & $\begin{array}{l}5.783^{* *} \\
(2.44)\end{array}$ & $\begin{array}{l}0.156 \\
(0.07)\end{array}$ & $\begin{array}{c}5.452^{* * *} \\
(2.75)\end{array}$ & $\begin{array}{l}3.500^{* *} \\
(2.04)\end{array}$ \\
\hline $\begin{array}{c}\text { The Cubic } \\
\text { Term of } \\
\text { Environmenta } \\
\text { 1 Regulation }\end{array}$ & $\begin{array}{c}-1.657^{* *} \\
(-2.37)\end{array}$ & $\begin{array}{l}-0.413 \\
(-0.48)\end{array}$ & $\begin{array}{c}-3.175^{* *} \\
(-2.49)\end{array}$ & $\begin{array}{l}-0.100 \\
(-0.10)\end{array}$ & $\begin{array}{c}-2.425^{* *} \\
(-2.23)\end{array}$ & $\begin{array}{c}-1.708^{* *} \\
(-2.05)\end{array}$ \\
\hline $\begin{array}{c}\text { Constant } \\
\text { Term }\end{array}$ & $\begin{array}{c}2.819^{* * *} \\
(8.02) \\
\end{array}$ & $\begin{array}{c}1.679^{* * *} \\
(4.12) \\
\end{array}$ & $\begin{array}{c}1.839 * * * \\
(4.56) \\
\end{array}$ & $\begin{array}{l}1.084^{*} \\
(1.86) \\
\end{array}$ & $\begin{array}{c}2.442^{* * *} \\
(7.64) \\
\end{array}$ & $\begin{array}{c}3.125^{* * *} \\
(9.62) \\
\end{array}$ \\
\hline $\begin{array}{l}\text { Number of } \\
\text { Sections }\end{array}$ & 98 & 99 & 83 & 49 & 104 & 127 \\
\hline $\begin{array}{l}\text { Observed } \\
\text { Value }\end{array}$ & 980 & 990 & 830 & 490 & 1040 & 1270 \\
\hline Hausman Test & $\begin{array}{c}47.68 \\
{[0.0000]}\end{array}$ & $\begin{array}{c}35.20 \\
{[0.0001]}\end{array}$ & $\begin{array}{c}42.66 \\
{[0.0000]}\end{array}$ & $\begin{array}{c}53.59 \\
{[0.0000]}\end{array}$ & $\begin{array}{c}58.10 \\
{[0.0000]}\end{array}$ & $\begin{array}{c}53.78 \\
{[0.0000]}\end{array}$ \\
\hline
\end{tabular}

Notes: ${ }^{* *}, * *$, and ${ }^{*}$ respectively represent the evaluation values being significant at the level of $1 \%, 5 \%$, and $10 \%$. The values in the round brackets are $t$ statistical amounts under the robust standard errors, and the values in the square brackets are $p$-values.

Table 4 reports the evaluation results of a fixed effect model analyzed with two different types of classification, namely, different areas and different scales. Through a comprehensive observation of Table 4, it can be determined that, although the influencing direction of each variable on the urban land utilization efficiency is almost in line with the total samples', there are still differences in the coefficients and the significance of industry clustering and industry specialization.

At first, there are regional differences in the influence of industry clustering on the urban land utilization efficiency. Specifically, although, theoretically speaking, there should be a significant inverted U-shaped relation in the eastern area, the critical value is 53.709. Although the quadratic term coefficients of the central and western areas are negative, they are statistically non-significant. 
The results above indicate that the enhancement of appropriate industry clustering will obviously facilitate the improvement of urban land utilization efficiency. Moreover, for central and western areas, active advancement of industry clustering is an effective approach to improve the land utilization efficiency.

Secondly, there are regional differences in the influence of industry specialization on urban land utilization efficiency. To begin with, there are significant differences in the influencing coefficient of different cities' industry specializations on the urban land utility efficiency from different areas. In detail, western coefficients are the largest and eastern coefficients are the smallest. Subsequently, from the perspective of significance, the relation between industrial specialization and urban land use efficiency in eastern areas are statistically non-significant, while in central and western areas both are significant at the level of $1 \%$. In other words, industry professional development plays a relatively important role in improving the land utilization efficiency of cities in central and western areas. However, it will not be the same case for cites in eastern areas. This also shows that, for cities in eastern areas, it will be more conducive to land utilization efficiency improvement via enhancing diversified industry development.

Thirdly, from the perspective of the scale levels of cities, although, theoretically speaking, there is an inverted U-shaped relation between industry clustering and the urban land utilization efficiency, the quadratic term coefficients of medium-sized cities are not significant, which manifests that active enhancement of the industry clustering of medium-sized cities will more effectively promote the improvement of the urban land utilization efficiency. Based on the values of coefficients, the inverted U-shape's inflection point of small-sized cities is the smallest, which indicates that, at present, the carrying capacity in terms of the industry clustering degree of small-sized cities in China is limited, and this situation further leads to an early arrival of the inflection point of the increasing effect of urban industry clustering on the urban land utilization efficiency.

Fourthly, speaking of the industry specialization, there are relatively large differences among cities at the three scale levels. Namely, the small-sized cities possess the largest coefficients ${ }^{\oplus}$, and they are significant at the level of $1 \%$, which indicates that the improvement of industry specialization of small-sized cities imposes a more significant improving effect on the land utilization efficiency, whereas, for large-sized cities, it is statistically non-significant compared with other city sizes, which manifests that it will be more beneficial to improving the land utilization efficiency of large-sized cities via facilitating diversified industry development.

\subsection{Robustness Test}

In order to test the robustness of the evaluation results, a further measurement of the land utilization efficiency, irrespective of environmental loss, has been exercised. Meanwhile, the total samples have been re-evaluated. Table 5 reports the evaluation results of the robustness test, and the results of Tables 4 and 5 are basically consistent, which means, theoretically speaking, there is a non-linear relation between industry clustering and urban land utilization efficiency. However, the quadratic term coefficients are relatively small and non-significant, which partly indicates that the current diseconomies of industrial agglomeration mainly present as environmental loss. At the same time, industry specialization is conducive to improving urban land utilization efficiency. In

\footnotetext{
(4) Coefficients here refer to the influencing coefficient of different cities' industry specialization on the urban land utility efficiency.
} 
addition, the influencing directions of the infrastructure situation, urbanization level, and environmental regulation on land utilization efficiency are basically consistent, while there are still some differences in terms of the variations in coefficients, and the evaluation of the infrastructure situation is not significant.

Table 5. Robustness test results of the total samples.

\begin{tabular}{|c|c|c|c|c|}
\hline $\begin{array}{l}\text { Explaining } \\
\text { Variables }\end{array}$ & (1) & $(2)$ & (3) & (4) \\
\hline $\begin{array}{l}\text { Industry } \\
\text { Clustering }\end{array}$ & $\begin{array}{c}0.117^{* * *} \\
(4.39)\end{array}$ & & $\begin{array}{c}0.112^{* * *} \\
(4.18)\end{array}$ & $\begin{array}{c}0.0991^{* * *} \\
(3.55)\end{array}$ \\
\hline $\begin{array}{l}\text { The Quadratic } \\
\text { Term of Industry } \\
\text { Clustering }\end{array}$ & $\begin{array}{c}-0.00112 \\
(-1.60)\end{array}$ & & $\begin{array}{c}-0.00103 \\
(-1.48)\end{array}$ & $\begin{array}{c}-0.00106 \\
(-1.50)\end{array}$ \\
\hline $\begin{array}{c}\text { Industry } \\
\text { specialization }\end{array}$ & & $\begin{array}{l}1.417^{* *} \\
(2.41)\end{array}$ & $\begin{array}{l}1.088^{*} \\
(1.85)\end{array}$ & $\begin{array}{c}1.302^{* *} \\
(2.23)\end{array}$ \\
\hline $\begin{array}{c}\text { Infrastructure } \\
\text { Situation }\end{array}$ & & & & $\begin{array}{c}0.00208 \\
(0.33)\end{array}$ \\
\hline $\begin{array}{c}\text { Urbanization } \\
\text { Level }\end{array}$ & & & & $\begin{array}{c}0.264^{* * *} \\
(6.75)\end{array}$ \\
\hline $\begin{array}{c}\text { Environmental } \\
\text { Regulation }\end{array}$ & & & & $\begin{array}{c}-3.022^{* * *} \\
(-4.88) \\
\end{array}$ \\
\hline $\begin{array}{c}\text { The Quadratic } \\
\text { Term of } \\
\text { Environmental } \\
\text { Regulation }\end{array}$ & & & & $\begin{array}{c}4.967^{* * *} \\
(4.58)\end{array}$ \\
\hline $\begin{array}{c}\text { The Cubic Term } \\
\text { of Environmental } \\
\text { Regulation }\end{array}$ & & & & $\begin{array}{c}-2.233^{* * *} \\
(-4.43)\end{array}$ \\
\hline Constant Term & $\begin{array}{l}3.222^{* * *} \\
(32.15)\end{array}$ & $\begin{array}{l}3.326^{* * *} \\
(21.78)\end{array}$ & $\begin{array}{l}2.962^{* * *} \\
(17.16)\end{array}$ & $\begin{array}{l}2.997^{* * *} \\
(14.02)\end{array}$ \\
\hline $\begin{array}{c}\text { Number of } \\
\text { Sections }\end{array}$ & 280 & 280 & 280 & 280 \\
\hline Observed Value & 2800 & 2800 & 2800 & 2800 \\
\hline
\end{tabular}

Notes: ${ }^{* *},{ }^{* *}$, and ${ }^{*}$ respectively represent the evaluation values being significant at the level of $1 \%, 5 \%$, and $10 \%$. The values in the round brackets are the $t$ statistical amount under the robust standard errors.

\section{Conclusions}

In order to analyze the relation between industry clustering, industry specialization, and the urban land utilization efficiency with the panel data from 280 prefecture-level cities from 2003 to 2013, this paper has taken environmental loss into consideration in the land evaluation system and adopted the SBM model to evaluate and measure the land utilization efficiency of each prefecture-level city. In conclusion, the prefecture-level cities in China present the following features of land utilization: (1) Generally speaking, the land utilization efficiency of the prefecture-level cities in China are relatively low, and the average value during the researching period is only 0.349 . Furthermore, theoretically speaking, there is still $65.1 \%$ land-saving potential in prefecture-level cities in China; (2) From the perspective of sequential characteristics, the land 
utilization efficiency of the prefecture-level cities in China from 2003 to 2013 generally presents, first, a decreasing trend, and then an increase. Moreover, the minimum points of the utilization efficiency value all appear in 2005, which indicates that there is a general momentum of gradual improvement in terms of land utilization efficiency; (3) Geographically speaking, the land utilization efficiency of the prefecture-level cities in China presents a "depression in the center" phenomenon, which means the land use efficiency of eastern cities is larger than central and western cities, and the land utilization efficiency of western cities is larger than the central cities; (4) The difference of the land utilization efficiency under environmental restrictions among Chinese cities possesses a narrowing trend. Moreover, the major contribution of the difference comes from the internal regional difference, which indicates that the land utilization efficiency among cities does not present a "club" trend. Meanwhile, the contribution of the internal difference of central cities to the total difference presents an increasing trend; and (5) from the perspective of the scale level of cities, small-sized cities possess significant advantages in terms of land utilization efficiency and, generally speaking, there is a U-shaped relation between the scale level of a city and the land utilization efficiency.

From a general point of view, theoretically speaking, there is an inverted U-shaped relation between industry clustering and urban land utilization efficiency. However, only Dongguan and Foshan show industry clustering levels that exceed the critical value in 2013, which indicates that, for the majority of cities, industry clustering can significantly improve the land utilization efficiency. In addition, industry professionalized development will significantly increase the urban land utilization efficiency. From the perspective of different areas, the industry professional level improvement of central and western areas imposes a more significant increasing influence on the urban utilization efficiency. Although, from the theoretical aspect, for eastern areas, there is an inverted U-shaped relation between industry clustering and the urban land utilization efficiency; there is no city whose efficiency value has exceeded the critical value. From the perspective of the scales of cities, the industry professional level improvement of small- and medium-sized cities imposes a more significant increasing influence on the urban utilization efficiency. Additionally, complete infrastructure and active enhancement of new-style and human-centered urbanization will be substantially conducive to improving the urban land utilization efficiency. Meanwhile, there is an inverted $\mathrm{N}$-shaped relation between environmental regulation and the land utilization efficiency.

Scientific planning and designing of urban land is an essential approach for improving the land utilization efficiency. Furthermore, the planning of industry land is an important part of the land utilization planning. Based on the research conclusions of this paper, there are several findings and suggestions, as follows: To begin with, it is vital to effectively link land utilization planning and industry planning. For most cities, it is necessary for the land utilization planning to lead the industry professional development and actively facilitate the developmental strategy of "one city, one industry", which embodies the idea of specially developing one unique, advantageous industry in one city. Meanwhile, for eastern areas and large-sized cities, it is necessary to make land utilization planning guide diversified industry development. In conclusion, via the convergent development of industry and cities, led by land utilization planning and through increasing industry clustering intensity, land utilization efficiency of Chinese cities will be further improved.

Acknowledgments: This research was financially supported by the National Social Science Foundation (15BJL053) and the Fundamental Research Funds for the Central Universities (2015AD005).

Author Contributions: The manuscript was collaboratively written by Zhangsheng Liu and 
Yuanyuan Gong. Deyong Song, Dongfang Li contributed to designing the study and interpreting the results, and also offered suggestions on the final revision. All authors have read and approved the final manuscript.

Conflicts of Interest: The authors declare no conflict of interest.

\section{References}

1. Liu Yansui, Deng Xusheng, Ganhong. Urban Land Use Situation and Opitimization Strategy in China[J]. Journal of Chongqing Jianzhu University, 2005, 27(3):1-4.

2. Chen Jianjun, Hu Chenguang. Agglomeration Effect of Industry Agglomeration: An Example based on Theoretical and Empirical Analysis of the Sub-Area in Yangtze River delta[J]. Management World, 2008(06):68-83.

3. Liu Xiping, Song Deyong. Influence of Urban industrial Agglomeration on Urban Environment[J]. Urban Problems, 2013(03):9-15.

4. Shi Fu, Shen Kunrong. Government Invention, Economic Agglomeration and Energy Efficiency[J]. Management World, 2013(10):6-18.

5. Fan Jianyong, Feng Meng, Li Fangwen. Industrial Agglomeration and Enterprise Total Factor Productivity[J]. The Journal of World Economy, 2014(05):51-73.

6. Yu Meizhen, Lin Zhifan. Can Industrial Specialization Boost City Economic Growth?--Empirical Research Based on Panel Data of 21 Cities of Guangdong Province [J]. Industrial Economic Reviews, 2014(05):139-148.

7. Zheng Xinqi, Wang Xiaoming. DEA Analysis for the Efficiency of Land Use Structure in Cities and Towns[J]. China Land Sciences, 2004, 18(2):34-39.

8. Song Ge, Gao Nan. Economic Benefit Analysis of Urban Land Utilization Based on DEA Method--A Case of Harbin City [J]. Scientia Geographica Sinica[J]. 2008, 28(2):185-188.

9. Wu Dewen, Mao Hanying, Zhang Xiaolei, et al. China Urban Land Use Efficiency Evaluation[J]. Acta Geographica Sinica[J]. 2011, 66(8):1111-1121.

10. Wang Xirui, Xu Shi, Yang Xingdian, et al. Analysis on Spatial and Temporal Difference of Construction Land Use Efficiency and Total Factor Productivity in Jiangsu Province[J]. China Land Sciences, 2015(5):77-83.

11. Du Guanyin,Cai Yunlong.Technical Efficiency of Built-up Land in China's Economic Growth during 1997-2007[J]. Progress in Geography,2010,29(6):693-700.

12. Liang Liutao, Zhao Qingliang, Chen Cong. Analysis on the Characters of Spatial Disparity of Urban Land Use Efficiency and Its Optimization in China[J]. China Land Sciences, 2013(7):48-54.

13. Lin Jian, Ma Xun. Land Use Efficiency Measurement of China's Urban Agglomeration[J]. Urban Problems, 2014(05):9-14.

14. Yang Haiquan, Hu Yi,Wang Qiuxiang. Evaluation of Land Use Efficiency in Three Major Urban Agglomerations of China in 2001-2012[J].Scientia Geographica Sinica,2015, 35(9):1095-1100.

15. Zhang Miao, Gan Chenlin,Chen Yin Rong.Carbon Emission Efficiency Analysis and Low Carbon Optimization for Urban Land Intensive Use Based on SBM Model[J].China Land Sciences,2016,30(3): 
$37-45$.

16. Li Hengji, Chen Xingpeng, Xu Xinyu, et al. Evolutionary Characteristics of Urban Efficiency in Gansu Province Based on the Super Efficiency DEA Model[J].Journal of Lanzhou University (Natural Sciences),2012, 48(5):35-40.

17. Zhang Zhihui.Study of Chinese Urban Land Use Efficiency[J].The Journal of Quantitative \& Technical Economics, 2014(7):134-149.

18. Hailu A, Veeman T S. Environmentally Sensitive Productivity Analysis of the Canadian Pulp and Paper Industry, 1959-1994: An Input Distance Function Approach[J]. Journal of Environmental Economics and Management, 2000, 40(3): 251-274.

19. Färe R, Grosskopf S, Lovell C A K, et al. Multilateral Productivity Comparisons When Some Outputs are Undesirable: A Nonparametric Approach[J]. The Review of Economics and Statistics, 1989: 90-98.

20. Färe R, Grosskopf S. Modeling Undesirable Factors in Efficiency Evaluation: Comment[J]. European Journal of Operational Research, 2004, 157(1): 242-245.

21. Lindmark M, Vikström P. Global Convergence in Productivity--A Distance Function Approach to Technological Progress and Efficiency Improvements[C]//Paper for the Conference Catching-Up Growth and Technology Transfers in Asia and Western Europe, Groningen. 2003: 17-18.

22. Chung Y H, Färe R, Grosskopf S. Productivity and Undesirable Outputs: A Directional Distance Function Approach[J]. Journal of Environmental Management, 1997, 51(3): 229-240.

23. Tone K. A Slacks-Based Measure of Efficiency in Data Envelopment Analysis[J]. European Journal of Operational Research, 2001, 130(3): 498-509.

24. Zhang Jun, Wu Guiying, Zhang Jipeng. The Estimation of China's Provincial Capital Stock: 1952-2000 [J].Economic Research Journal, 2004(10):35-44.

25. Managi S, Kaneko S. Environmental Productivity in China[J]. Economics Bulletin, 2004, 17(2):1-10.

26. Tu Zhengge.Rural Land System, Land Fragmentation and Farmer's Income Inequality[J].Economic Research Journal, 2008 (2) :93-105.

27. Wang Bing, Wu Yanrui, Yan Pengfei.Environmental Efficiency and Environmental Total Factor Productivity Growth in China's Regional Economies[J].Economic Research Journal, 2014(9): 95-109.

28. Zhang Wei,Zhu Qigui,Li Hanwen. Energy Use, Carbon Emission and China's Total Factor Carbon Emission Reduction Efficiency[J].Economic Research Journal, 2013(10):138-150.

29. Duranton G, Puga D. Nursery Cities: Urban Diversity, Process Innovation, and the Life Cycle of Products[J]. American Economic Review, 2001: 1454-1477.

30. Sun Xuemin,Wang Jie. Effect of Environmental Regulation on Chinese Enterprise Size Distribution [J].China Industrial Economics,2014 (12): 44-56.

31. Wang Jie, Liu Bin.Environmental Regulation and Enterprises' TFP - - An Empirical Analysis Based on China's Industrial Enterprises Data [J].China Industrial Economics, 2014(3):44-56. 